\title{
Fitotoxicidade de efluente da indústria cervejeira em sementes de Lactuca sativa L.
}

\author{
Lucas Oliveira Viana ${ }^{\prime}$, Kelly Geronazzo Martins' ${ }^{I}$ Kely Viviane de Souza', Erivelton Cesar Stroparo ${ }^{I}$ \\ هlucaslov@hotmail.com
}

1. Universidade Estadual do Centro-Oeste do Paraná - Campus Irati, Rodovia PRT 153, Km 338, Irati, Brasil.

\section{Histórico do Artigo:}

Recebido em:21 de agosto de 2017

Aceito: 22 de novembro de 2017

Publicado: 5 de janeiro de 2018

Resumo:0s efluentes gerados na indústria cervejeira apresentam elevada carga orgânica, fazendo-se necessário um alto grau de tratamento da água no processo, antes de retorná-la à natureza. 0 adequado tratamento evita ou reduz danos ao ambiente e consequentemente ao homem. Nesse contexto o objetivo do presente estudo foi avaliar a fitotoxicidade do efluente oriundo da indústria cervejeira mediante avaliação do desenvolvimento da radícula, da porcentagem de germinação e do índice de germinação de sementes de Lactuca sativa L. (alface) bem como a correlação dessas variáveis com a Cor e a DQO do efluente bruto e tratado. Para tanto foram avaliadas 540 sementes de alface divididas em 4 concentrações, um controle positivo e outro negativo, cada uma das quais representadas por 3 placas de Petri (repetições) com 15 sementes cada. As sementes foram incubadas em estufa DB0 na ausência de luz, a temperatura constante de $22^{\circ} \mathrm{C} \pm 2^{\circ} \mathrm{C}$, por 120 horas. Ao final do período de exposição os resultados demostraram que não há diferença estatística entre a $\mathrm{CE}_{50}$, o crescimento da radícula e 0 índice de germinação do efluente bruto e tratado, sendo que o índice de alongamento da raiz demostrou inibição significativa segundo o método tanto para o efluente tratado como para o bruto. Uma vez que a porcentagem de germinação e o crescimento da radícula apontaram correlação significativa com a cor e a DQ0, é possível concluir a ineficiência do tratamento por processo oxidativo avançado no efluente estudado.

Palavras-chave:Toxicidade, $\mathrm{CE}_{50}$, Águas Residuárias.

\section{Phytotoxicity of beer industry effluents in Lactuca sativa L. seeds}

\begin{abstract}
The effluent generated in the beverage industry is rich in organic load, requiring a high degree of treatment of the process water, before returning it to nature. In this context, the main objective of this study is to verify was to evaluate the phytotoxicity of effluent from the beverage industry by root elongation, and seed germination of Lactuca sativa L. (lettuce) as well as the correlation of these variables with the Color and COD of the raw and treated effluent. The seed germination and root elongation of 540 lettuce seeds divided into 4 concentrations, one positive control and one negative control, each of which was represented by 3 Petri dishes (replicates) with 15 seeds in each plate were evaluated. The seeds were incubated in the absence of light at a constant temperature of $22 \pm 2{ }^{\circ} \mathrm{C}$ for 120 hours. At the end of the exposure period, the results showed that there was no statistical difference between the $\mathrm{EC}_{50}$ root growth and the germination index of the treated and treated effluent, and the root elongation index showed significant inhibition according to the method for both the effluent Treated as for the brute. Since the percentage of germination and root growth showed a significant correlation with color and COD, it is possible to conclude the treatment inefficiency of the treatment by advanced oxidative process in the studied effluent.
\end{abstract}

Keywords:Toxicity, $\mathrm{EC}_{50}$, Wastewater. 


\title{
Fitotoxicidad de efluente de la industria cervecera en semillas de Lactuca sativa L.
}

\begin{abstract}
Resumen:Los efluentes derivados de la industria cervecer apresentan una alta carga orgánica, lo que hace necesario un intenso tratamiento de las aguas residuales antes de regresar a la naturaleza. El tratamiento correcto y adecuadodel agua reduce los daños medio ambientales y a la populación. En este contexto el objetivo de este estudio fue la evalución de lo desenvolvimiento de la raíz, de la porcentaje y índice de germinación de semillas de Lactuca sativa L. (Lechuga) y también la comparación de las variables com el color y la DQ0 del efluente antes y después del tratamiento. Por lo tanto fueron evaluadas 540 semillas de lechugas divididas em 4 concentraciones, un control positivo y otro negativo, cada uno de ellas representadas por 3 platos petri (repetición) con 15 semillas cada uno. Las semillas fueron incubadas en una estufa DB0 junto a la ausencia de la luz, regulada a $22 \pm 2{ }^{\circ} \mathrm{C}$, por 120 horas. Al final del estudio de exposición los resultados muestran que no hay diferencia estadística entre la $\mathrm{CE}_{50}$, elcrecimiento de laraíz y el índice de germinacióndel efluente antes y despúes del tratamiento, siendo que el índice alargamiento de la raíz demuestra inibición significativa segun el metodo tanto para el efluente tratado cuanto para el sin tratamiento. Una vez que la porcentaje de germinación y el crecimiento de la raíz apuntaron correlación significativa con el color y la DQ0, es posible concluir la ineficiencia del tratamiento por proceso oxidativo avanzado en el efluente estudiado.
\end{abstract}

Palabras clave:Toxicidad, $\mathrm{CE}_{50}$, Efluentes.

\section{INTRODUÇÃO}

0 Brasil conquistou em anos recentes a terceira posição na lista dos maiores consumidores mundiais de cervejas e refrigerantes em 2015 segundo o banco nacional do desenvolvimento. Em 2011 alcançou um faturamento de R\$ 20 bilhões, empregando direta e indiretamente mais de 150 mil pessoas, destacando-se com a maior arrecadação de tributos indiretos entre todos os setores da economia (BNDES, 2016).

Na fabricação da cerveja a fermentação da cevada é a principal etapa do processo e consiste na conversão em álcool dos açúcares presentes nos grãos da mesma. Após a fermentação são realizadas etapas de tratamento que podem variar muito conforme o tipo da cerveja, pois, são elas que irão conferir as características organolépticas (sabor, odor, textura) desejadas no produto final (SINDICERV, 2012), a geração de efluentes nesses processos é significativa, pois a cada litro de cerveja produzida são gerados cerca de 6 litros de efluente com alta concentração de matéria orgânica (SANTOS; RIBEIR0, 2005).

Independentemente da forma de tratamento dos efluentes gerados na indústria cervejeira faz-se necessário o monitoramento dos mesmos, tanto mediante avaliações de parâmetros químicos e físicos, como, especialmente, pela avaliação de toxicidade aguda ou crônica em relação à organismos-teste. 
Ensaios ecotoxicológicos são indispensáveis na disposição de qualquer tipo de efluente em corpos hídricos uma vez que os parâmetros físicos e químicos usualmente estabelecidos, como carbono orgânico total (COT), metais, sólidos, demanda química de oxigênio (DQO) e demanda biológica de oxigênio (DB0), entre outros, são insuficientes para discriminar compostos que podem afetar os corpos receptores, assim, constituem-se insuficientes para a avaliação do risco ambiental dos contaminantes (PIRES; CHAPARR0, 2010).

Contudo os ensaios ecotoxicológicos não substituem as análises físico-químicas, que identificam e quantificam contaminantes, e sim avaliam os efeitos biológicos destes em corpos hídricos receptores, de forma que ambos se complementam mutuamente (PIRES; CHAPARR0, 2010).

Particularmente, os testes de toxicidade com espécies de plantas vasculares (EC, 2007) permitem a avaliação dos efeitos adversos sobre a germinação de sementes e desenvolvimento das plântulas durante os primeiros dias de crescimento (DUTKA, 1989; LEWIS, 1995). Estes testes permitem avaliar potencialmente os efeitos negativos sobre as plantas causadas pela descarga de efluentes ao longo do solo. Avaliações de fitotoxicidade já foram usadas para caracterizar os impactos adversos em diversos tipos de águas residuárias (PASCUAL et al., 1997).

Nesse contexto a alface (Lactuca sativa L.) é um dos organismos indicados para análises ecotoxicológicas, especialmente por representar um vegetal amplamente cultivado, utilizado em grande escala na alimentação humana e animal e, vulnerável a contaminações por irrigação contendo águas residuárias (GIORGETTI et al., 2011; YOUNG et al., 2011).

Segundo PELEGRINI et al. (2009), as sementes de alface constituem excelentes organismos para bioensaios de toxicidade de efluentes tratados pelos mais diversos processos, pois, apresentam alta sensibilidade ao estresse químico, bem como rápidas germinação e reidratação, garantindo a reprodutibilidade do ensaio. Diante desse cenário, evidencia-se a importância de estudos que caracterizem de forma química e toxicológica o efluente de indústrias cervejeiras no Brasil, assim os objetivos do presente estudo foram: I Determinar a Demanda Química de 0xigênio (DB0), a cor aparente e verdadeira do efluente oriundo da produção de cerveja; II Avaliar a fitotoxicidade do efluente da indústria cervejeira mediante 0 desenvolvimento da raiz e da porcentagem de germinação de sementes de Lactuca sativa L.; III 
Correlacionar o crescimento da raiz e a germinação das sementes de Lactuca sativa L com parâmetros físico-químicos.

\section{MATERIAL E MÉTODOS}

Inicialmente avaliou-se dois parâmetros físico químicos: a demanda química de oxigênio $(\mathrm{DQ} 0)$ e a cor verdadeira dos efluentes bruto (após tratamento da empresa) e tratado (após tratamento por processo oxidativo avançado), com o intuito de comparar a eficiência do tratamento pela Análise de Variancia (ANOVA) em conjunto com o teste de Tukey a 95\% de confiança

A determinação da DQ0 feita para verificar a eficiência do processo aplicado ao efluente, realizado de acordo com a metodologia padrão (APHA-5220D), fundamentada em um processo de digestão em tubo fechado seguida de determinação colorimétrica em 600 nm(APHA, 2012).

A cor verdadeira foi determinada de acordo com absorbância no comprimento de onda máximo na região do visível $(455 \mathrm{~nm})$ após centrifugação a $3500 \mathrm{rpm}$, utilizando espectrofotômetro marca HACH, modelo DR6000.

Para avaliar a sensibilidade das sementes frente a um composto tóxico conhecido, foi utilizado como controle positivo $(+)$ soluções de sulfato de zinco e, para verificar a viabilidade do lote empregou-se como controle negativo (-) água destilada.

0 experimento com as amostras controle foram realizados no mesmo padrão que os ensaios com as soluções-teste.

A Montagem dos experimentos ocorreu em placas de petri, previamente limpas e secas colocou-se papéis-filtro qualitativos de $9 \mathrm{~cm}$ de diâmetro e $4 \mathrm{~mL}$ do efluente bruto e tratado a serem avaliados. Com auxílio de uma pinça serão distribuídas uniformemente 15 sementes em cada placa. Para cada amostra e controle os experimentos foram realizados em triplicata.

Para manter a umidade dentro das placas, usou-se pequenos chumaços de algodão, embebidos em água destilada. Posteriormente, as placas foram embrulhadas com papel filme plástico (para evitar a evaporação) e, incubadas (em estufa DB0) na ausência de luz, a temperatura constante de $22^{\circ} \mathrm{C} \pm 2{ }^{\circ} \mathrm{C}$, por 120 horas. Ao final do período de exposição 
contabilizou-se o número de sementes que germinaram em cada placa e destas foram medidos os alongamentos das raízes em centímetros.

Afim de legitimar o experimento, para validação do bioensaio, no mínimo 90\% das sementes da placa controle (-) devem germinar. Por sua vez, o coeficiente de variação (equação 1) de alongamento da raiz não deve ultrapassar 30\%. Caso contrário, os resultados devem ser considerados suspeitos e os testes repetidos com outro lote de sementes.

$$
\mathrm{CV}=\frac{S}{X} \times 100
$$

Onde, CV é o coeficiente de variação, S o desvio padrão e X a média.

0 número de sementes germinadas foi utilizado para o cálculo da germinação relativa (GR) (Equação 1) e da concentração da amostra responsável pelo efeito em $50 \%$ dos organismos testados $\left(\mathrm{CE}_{50}\right)$, os dados foram utilizados para os cálculos do índice de crescimento relativo (IAR) (Equação 2) e, a partir dos valores de GR e IAR determinou-se o índice de germinação (IG) (Equação 3).

$$
\begin{aligned}
& \mathrm{GR}=\frac{S G A}{S G C} \\
& \mathrm{IAR}=\frac{M A R A}{M A R C} \\
& \mathrm{IG}(\%)=\text { GR } \times \text { IAR } \times 100
\end{aligned}
$$

Em que, o SGA corresponde ao número de sementes germinadas na amostra, SGC o número de sementes germinadas no controle (-), MARA a média do alongamento da raiz na amostra e MARC a média do alongamento no controle (-).

Os valores de IAR serão classificados em três categorias de acordo com os efeitos tóxicos observados: 
- Inibição do alongamento da raiz (I): $0<x>0,8$;

- $\quad$ Não houve efeito significativo (NES): $0,8 \leq x \geq 1,2$;

- $\quad$ Estímulo do alongamento da raiz (E): $x>1,2$.

Onde $x$ é 0 valor obtido pela equação 2.

Os experimentos seguiram um delineamento inteiramente casualizado com três repetições. Objetivando aferir se existem diferenças entre o efluente bruto e o tratado, os dados foram submetidos ao teste $t$ student para amostras dependentes. Afim de comparar os dois tipos do efluente em conjunto com o controle optou-se pela análise de variância e o teste de Tukey.

Gerou-se modelos de regressão afim de estimar a $\mathrm{CE}_{50}$ (concentração da amostra responsável pelo efeito em $50 \%$ dos organismos testados).

Análise de redundância canônica foi efetuada para averiguar possíveis relações entre os parâmetros determinados nos ensaios físico-químicos e os índices toxicológicos IAR e $\mathrm{CE}_{50}$.

As premissas de gaussianidade de resíduo e homogeneidade de variância foram validadas pelos testes de Shapiro -Wilk e Bartlett. No caso da regressão, além da gaussianidade de resíduo a ausência de colinearidade foi discriminada pelo teste de DurbinWatson. 0 nível de significância para todos os testes será de $5 \%(\mathrm{p}<0,05)$.

\section{RESULTADOS E DISCUSSÃO}

\section{Determinação dos parâmetros físico-químicos}

Após o tratamento efetuado pela empresa os valores da Cor Aparente e Cor Verdadeira reduziram significativamente, porém os valores de DQ0 seguiram estatisticamente semelhantes (Gráfico 1).

Segundo Braile e Cavalcanti, 1993, a DQ0 presente no efluente da indústria brasileira de cerveja é elevada devido à alta carga orgânica do efluente, podendo variar de 1290 até 80000 . Nesse contexto, vale ressaltar que se faz necessário um alto grau de tratamento, exigindo uma 
combinação de processos para que a remoção dos poluentes seja eficiente (COSTA-FILHO et al., 2016), uma vez que a concentração de DQ0 tanto do efluente bruto como do tratado está acima do limite permitido (300 mg L ${ }^{-1}$ ) pela resolução n ${ }^{0} 70$ de 2009 do Conselho Estadual do Meio Ambiente (CEMA, 2009). Quanto à cor, não há inclusão de um padrão para emissão desse parâmetro de qualidade da água na resolução nº 430 de 2011 do CONAMA.

Gráfico 1 - Médias e quartis de efluente bruto e tratado oriundo da indústria cervejeira. Médias seguidas da mesma letra não diferem estatisticamente a $5 \%$ de significância pelo teste t para amostras dependentes.
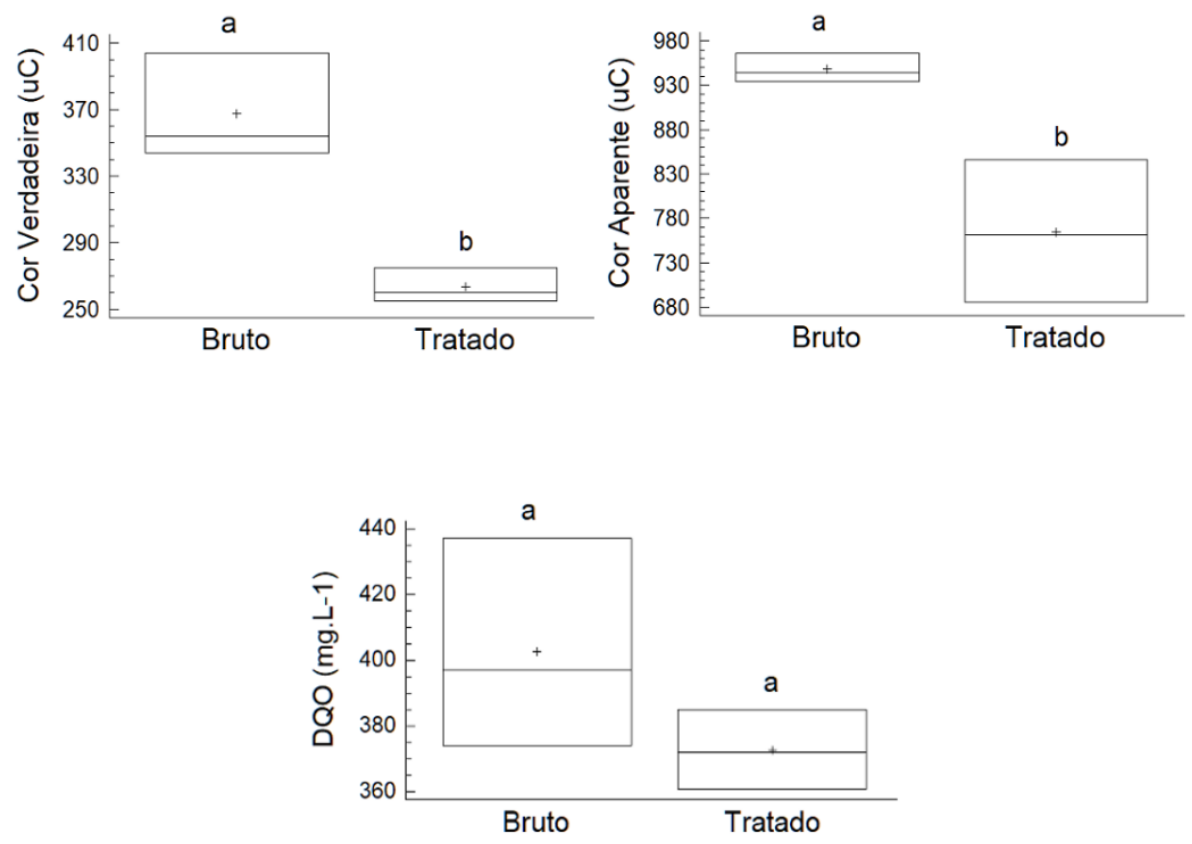

Fonte: Elaborado pelo autor.

\section{Toxicidades do efluente bruto e do tratado}

No presente estudo, o coeficiente de variação do comprimento da raiz na amostra controle foi de 5,35\% e germinaram 97\% das sementes, portanto, os critérios estabelecidos para validação do bioensaio foram aceitos.

Foi observado necrose em $37 \%$ das sementes expostas ao efluente bruto e, a $\mathrm{CE}_{50}$ para a germinação foi de $75 \pm 8,0 \%$. Para o efluente tratado foi observado necrose em $35 \%$ das 
sementes expostas ao efluente bruto e, a $\mathrm{CE}_{50}$ para a germinação foi de $74 \pm 5,9 \%$. Os valores elevados de $\mathrm{CE}_{50}$ refletem uma baixa toxicidade do efluente. Camargo (2012) verificou para os ensaios de toxicidade aguda com Daphiniasimilis $\mathrm{CE}_{50}$ igual a 41,67\%. Uma vez que o microcrustáceo é mais sensível à toxicidade que as sementes de alface (COSTA e OLIVI, 2008), é razoável considerar que os resultados de $\mathrm{CE}_{50}$ para um teste com microcrustáceos seriam menores que os apresentados.

Os efeitos do efluente bruto e do tratado sobre a germinação das sementes de L. sativa L.são apresentados na Gráfico 2.

Gráfico 2 - Índice de germinação (IG) de sementes de L. sativa L. após a exposição às diferentes amostras estudadas e 0 controle. Médias seguidas pela mesma letra não diferem significativamente entre si pelo teste de Tukey a 5\% de probabilidade de erro.

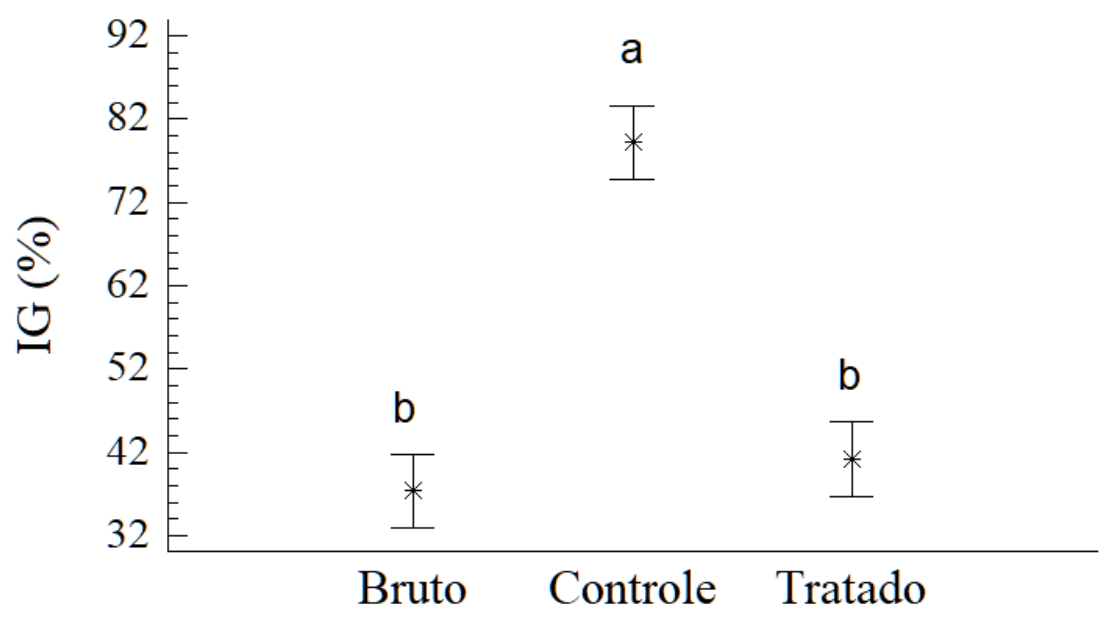

Fonte: Elaborado pelo autor.

Correlações do comprimento da raiz e da porcentagem de germinação com a DQO e Cor Aparente e Verdadeira.

A variável Cor Aparente e DQO (matriz de preditórias) correlacionaram significativamente com o crescimento da raiz e com a porcentagem de germinação das sementes (matriz de variáveis respostas). De forma geral a análise de redundância demostrou que as duas matrizes apresentam uma correlação de 0,95 para o primeiro eixo e 0,87 para 0 
segundo (Gráfico 3). Tais correlações foram determinadas como significativas mediante o teste de Monte Carlo (999 permutações).

Tanto a Cor Verdadeira como a DQ0 correlacionaram negativamente com o comprimento da raiz e com a porcentagem de germinação. Young et al. (2011) em estudo com efluente da indústria de cereais verificaram efeitos no desenvolvimento de plantas vasculares. Diversos estudos comprovam que ensaios com sementes de L. sativa L.permitem a detecção de efeitos biológicos de substâncias tóxicas contidas em efluentes, conforme indicado por Walsh et al. (1991).

Gráfico 3 - Análise de redundância de parâmetros da qualidade da água e indicadores de toxicidade de efluentes da indústria cervejeira.

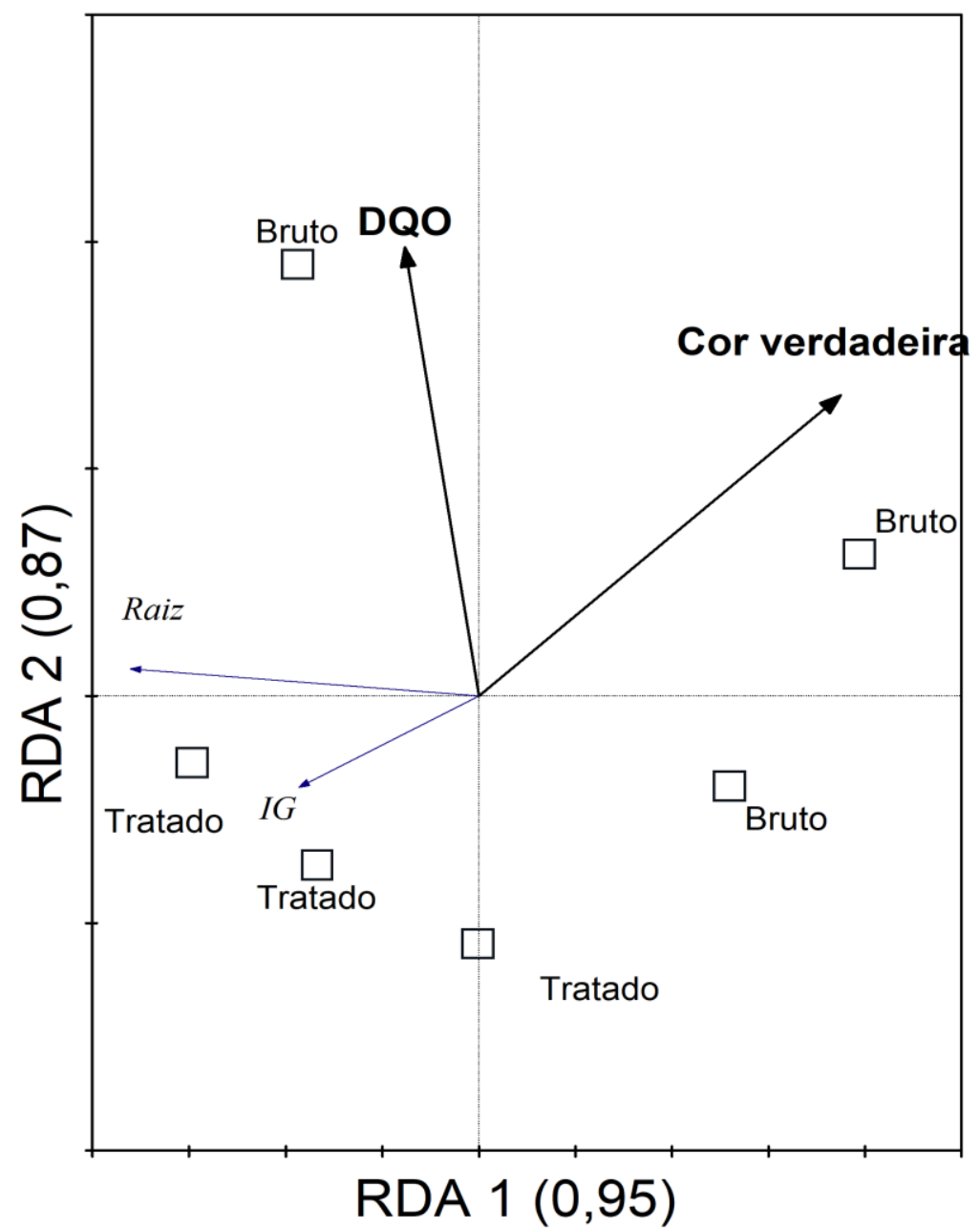

Fonte: Elaborado pelo autor. 


\section{CONCLUSÃ0}

De forma geral os resultados do presente estudo demostram que não há efeito significativo do tratamento de efluente usado pela empresa no índice de germinação, ilustrando o efeito perturbador tanto do efluente bruto como do tratado ao ecossistema caso sementes de plantas vasculares fossem imediatamente irrigadas com o mesmo ou caso este seja lançado às margens de corpos d'águas próximas as comunidades de plantas.

\section{REFERÊNCIAS BIBLIOGRÁFICAS}

APHA - AMERICAN PUBLIC HEALTH ASSOCIATION. Standard Methods for the examination of Water and Wastewater. 22.ed. Washington: American Public Health Association, 2012.

BANCO NACIONAL D0 DESENVOLVIMENTO BNDES, (0rg.). 0 setor de bebidas no Brasil. 2016. Disponível em: <https://web.bndes.gov.br/bib/jspui /bitstream/1408/3462/1/BS 400 setor de bebidas no Brasil_P.pdf $>$. Acesso em: $31 / 10 / 2017$

BRAILE, P.M.; CAVALCANTI, J.E.W.A. Manual de tratamento de águas residuárias industriais. São Paulo: CETESB, 1993.

CAMARG0, J.B.D.A.; ARAÚJ0, G.S.; CRUZ. A.C.F.; FONSECA, T.G.; ABESSA, D.M.S. Use, development and improvement in the protocol of whole-sediment Toxicity Identication and Evaluation using benthic copepods. Marine PollutionBulletin, 91(2): 511-517. 2012. Disponível em: http://www.sciencedi rect.com/science/article/pii/S0025326X14006833?via\%3Dihub. Acesso em 20/02/2017.

CONSELHO ESTADUAL DO MEI0 AMBIENTE- CEMA. Resolução nº70, de 11 de agosto de 2009. Dispõe sobre 0 licenciamento ambiental, estabelece condições e critérios e da outras providências, apara Empreendimentos Industriais. $\quad$ Disponível em: $\quad \mathrm{http} / /$ www.iap.pr.gov.br/arquivos/File/L egislação_ambienta/Legislação_estadual/RESOLUCOES/resolucao_cema_70_2009.pdf. Acesso em 29 de julho de 2017

CONSELHO NACIONAL DO MEIO AMBIENTE - CONAMA. Resolução no 430, de 13 de maio de 2011. Dispõe sobre as condições e padrões de lançamento de efluentes, complementa e altera a Resolução no 357, de 17 de março de 2005, do Conselho Nacional do Meio Ambiente-CONAMA. Disponível em: http://www.mma.gov.br/port/conama/legiabre.cfm?codlegi=646. Acesso em 29 de julho de 2017.

COSTA-FILHO, B. M.; SILVA, V. M.; SILVA, J. 0.; HORA M.; ANTONIO E. Coupling coagulation, flocculation and decantation with photo-Fenton process for treatment of industrial wastewater containing fipronil: 
Biodegradability and toxicity assessment. JournalOf Environmental Management, v. 174, p.71-78, 2016. Disponível em: http://www.sciencedirect.com/science/article/pii/ S0301479716301104?via\%3Dihub. Acesso em 21/02/2017.

COSTA, C.R.; OLIVI, P; BOTTA, C.M.R.; ESPINDOLA, E.L.G. A toxicidade em ambientes aquáticos: discussão e métodos de avaliação. Quimica. Nova, v. 31, n. 7, p. 1820-1830, 2008. Disponível em:

http://quimicanova.sbq.org.br/imagebank/pdf/Vol31No7_1820_37-RV07485.pdf. Acesso em 23/02/2017.

DUTKA, B.J., 1989. Short-Term Root Elongation Toxicity Bioassay. Methods for Toxicological Analysis of Waters, Wastewaters and Sediments. National Water Research Institute (NWRI), Environment Canada.

EC, 2007. Biological Test Method:Test for Measuring Emergence and Growth of Terrestrial Plants Exposed to Contaminants in Soil, EPS 1/RM/45. Environmental Technology Center, Environment Canada, Ottawa. Disponivel em: http://publications.gc.ca/collections/collection_2014/ec/En49-7-1-45-eng.pdf. Acesso em 30/01/2017.

GIORGETTI, L.; TALOUIZTE, H.; MERZOUKI, M.; CALTAVUTURO, L.; GERI, C.; FRASSINETTI, S. Genotoxicity evaluation of effluents from textile industries of the region Fez-Boulmane, Morocco: A case study. Ecotoxicology and Environmental Safety, $\quad$ n.74, $\quad$ p. $2275-2283, \quad 2011 . \quad$ Disponivelem: http://www.sciencedirect.com/science/article/pii/S0147651311002454. Acesso em 22/02/2017.

LEWIS, M.A. Algae and vascular plant tests. in: Rand, G. (Ed.), Aquatic Toxicology: Effects, Environmental Fate and Risk Assessment, Taylor e Francis Publishers, USA, p. 135-169. 1995.

PASCUAL, J.A.; AYUSO, M.; GARCIA, C.; HERNANDEZ, T. Characterization of urban wastes according to fertility and phytotoxicity parameters.Waste Management EResearch, p. 15,103-112. 1997. Disponível em: http://www.sciencedirect.com/science/article/pii/S0734242X96900671?via\%3Dihub. Acesso em 22/02/2017.

PELEGRINI, R.T.; BRIT0-PELEGRINI, N.N. de; PATERNIANI, J.E.S.; BROTA, G.A.; SANTOS, E.M.; SILVA, N.B., Ensaios biológicos com sementes para avaliar a redução da toxicidade do chorume tratado por processo fotoquímico. 2009. Disponível em: http://www.fipai.org.br/Minerva\%2006(03)\%2001.pdf. Acesso em 22/02/2017.

PIRES, E. C.; CHAPARR0, T. R. Toxicity evaluation as a tool to assess the performance of an anaerobic immobilized biomass reactor. DYNA, Medellín, v.77, n.164, p.284-391, 0ct. /Dec. 2010. Disponível em: http://www.scielo.org.co/pdf/dyna/v77n164/a28v77n164.pdf. Acesso em 22/02/2017.

SANTOS, M.S.; RIBEIR0, F.M. Cervejas e refrigerantes. São Paulo: CETESB, 2005.58p.http://www.cetesb.sp.gov.br>. Acesso em 23/02/2017.

SINDICATO NACIONAL DA INDÚSTRIA DA CERVEJA - SINDICERV,2012. Disponível em: www.sindicerv.com.br acesso em: 30 de jan. 2017.

YOUNG, B., J.; RIERA, N., I.; BEILY, M., E.; BRES, P. A.; CRESPO, D., C.; RONCO, A., E. Toxicity of the effluent from ananaerobic bioreactor treating cereal residues on Lactuca sativa. Ecotoxicology and Environmental Safety n. 76 p. 182-186. 2011. Disponível em: http://www.sciencedirect.com/sc ience/article/pii/S0147651311003125.

WALSH, G., WEBER, D., SIMON, T., BRASHERS, L., Toxicity test of effluents with marsh plants in water and sediment. Environmental ToxicologyandChemistry. 10, 517-525. 1991. Disponível em: http://onlinelibrary.wiley.com/ doi/10.1002/etc.5620100412/epdf?r3_referer=wol\&tracking_action=preview_click\&show_checkout=1\&purchase_refe rrer=www.google.com.brôpurchase_site_license=LICENSE_DENIED. acesso em: 30 de jan. 2017. 